\title{
Traduire
}

Une eutre perspective sur $r$ tatadciction

Revue française de la traduction

$240 \mid 2019$

Quand la politique s'en mêle

\section{Les voix de l'asile}

Enjeux politiques de l'interprétariat dans la procédure de demande d'asile

\section{Maxime Maréchal}

\section{Q OpenEdition}

\section{Journals}

Édition électronique

URL : http://journals.openedition.org/traduire/1696

DOI : 10.4000/traduire.1696

ISSN : 2272-9992

Éditeur

Société française des traducteurs

\section{Édition imprimée}

Date de publication : 20 juin 2019

Pagination : 69-79

ISSN : 0395-773X

\section{Référence électronique}

Maxime Maréchal, « Les voix de l'asile », Traduire [En ligne], 240 | 2019, mis en ligne le 29 juin 2019,

consulté le 06 février 2020. URL : http://journals.openedition.org/traduire/1696 ; DOI : 10.4000/

traduire.1696 


\section{Les voix de I'asile}

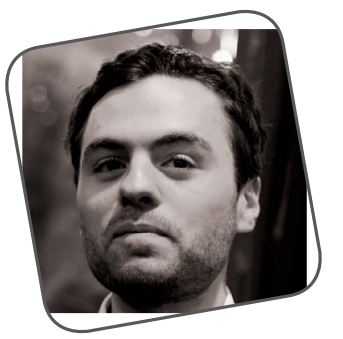

\section{Enjeux politiques de l'interprétariat dans la procédure de demande d'asile}

\section{Maxime Maréchal}

Demander l'asile politique en France est avant tout une question de motivation. Non seulement celle de poursuivre cette difficile entreprise, mais aussi au sens où il faut légitimer les raisons qui ont conduit à demander la protection de l'État. Dès son arrivée sur le territoire français, l'exilé devient en effet "demandeur» auprès de l'Office de protection des réfugiés et apatrides (OFPRA), éventuellement «requérant» auprès de la Cour nationale du droit d'asile (CNDA)': il est intégralement assigné au registre de la requête jusqu'au refus ou à l'accord de l'administration de l'asile. La décision de faire du demandeur un réfugié ou au contraire un débouté repose essentiellement sur la manière dont il aura, en la justifiant et en la défendant, présenté sa demande eu égard aux critères juridiques qui conditionnent l'obtention de la protection conventionnelle ${ }^{2}$. Cette question prend une importance particulière dans le contexte français actuel, où l'exposition médiatique, mais aussi les politiques de l'asile manifestent une suspicion généralisée envers les demandeurs, catégorisés en «vrais» ou «faux»réfugiés ${ }^{3}$.

1. L'Office de protection des réfugiés et des apatrides est un établissement public administratif qui statue sur les demandes d'asile et d'apatridie; la Cour nationale du droit d'asile juge quant à elle les demandes de recours aux rejets décidés par l'OFPRA.

2. Selon la convention de Genève de 1951, est reconnue comme réfugiée «toute personne craignant avec raison d'être persécutée du fait de sa race, de sa religion, de sa nationalité, de son appartenance à un certain groupe social ou de ses opinions politiques».

3. Sur cette question, voir Akoka, 2011. 
Aussi les moments d'interaction du demandeur avec les représentants de l'administration de l'asile (l'officier de protection de l'OFPRA comme la formation de jugement de la CNDA) revêtentils une importance capitale, puisque c'est là que le demandeur devra convaincre qu'il est un «vrai » réfugié.

Or ces interactions, dans plus de $90 \%$ des cas, exigent l'intervention d'un interprète. Tout demandeur peut en jouir gratuitement, selon des conditions définies juridiquement ${ }^{4}$.

L'interprète a ainsi le rôle fondamental de créer l'espace de communication permettant au demandeur de justifier son dossier et de convaincre des bonnes raisons qui le motivent. Cette fonction est d'autant plus déterminante qu'elle est au cœur du registre de la probation et de l'authentification qui conditionne l'acceptation ou le refus des demandes d'asile.

Mais cette interprétation s'exerce dans des conditions complexes. La multiplication des demandes a créé un besoin urgent d'interprètes en de nombreuses langues. De plus, l'interprète se trouve intégré à une relation fortement asymétrique, entre celui qui requiert et celui qui octroie, ce qui ne cesse de mettre en question son positionnement vis-à-vis des autres acteurs au sein du dispositif. Enfin, il doit communiquer des réalités entre des interlocuteurs d'univers culturels et sociaux parfois très éloignés.

En quoi le rôle de l'interprète dans la demande d'asile est-il rendu problématique par sa place institutionnelle? En quoi diffère-t-il radicalement de la simple fonction de transmission de messages qui lui est assignée? Quels sont alors les enjeux politiques de l'interprétation?

Voici les questions autour desquelles, sur la base d'observations ethnographiques effectuées à la CNDA et d'échanges (entretiens ou discussions informelles) avec des interprètes, je tenterai d'esquisser quelques pistes de réflexion.

\section{Un expert de la langue?}

Comparativement à l'interprétariat de conférence ou à celui effectué dans le cadre médical, l'interprétariat de service public (dont relève l'interprétation dans la procédure d'asile) a été

4. En dépit des discussions sur la dénomination de l'activité de l'interprète, j'emploierai ici aussi bien interprétariat (utilisé par les institutions) qu'interprétation. Par ailleurs, le terme «interprète» désignera aussi bien les femmes que les hommes qui exercent ce métier. 
peu étudié. C'est une branche assez récente des métiers de la traduction, qui n'est pas la plus prestigieuse ni la mieux rémunérée, mais qui présente, aux yeux de certains interprètes, des bénéfices spécifiques (intellectuels, humains...). Amené à établir la relation entre l'administration et un individu issu d'une minorité dont il peut faire lui-même partie (d'où son nom de community interpreting dans les pays anglo-saxons), l'interprète y pratique l'interprétation consécutive de dialogue. Ce secteur est ainsi caractérisé par une professionnalisation plus faible que dans les autres types d'interprétariat, par une forte asymétrie sociopolitique entre les personnes en dialogue, ainsi que par la charge émotionnelle et morale particulièrement conséquente des interactions.

\section{Les normes de l'interprétation}

À l'OFPRA et à la CNDA, l'interprétation est définie selon des normes juridiques et déontologiques qui doivent garantir sa conformité avec leur mission. Ce sont ces normes qui permettent de comprendre le rôle attribué à l'interprète dans le dispositif de l'asile, et la manière dont il le jove.

Ainsi, le Code de l'entrée et du séjour des étrangers et du droit d'asile (CESEDA) ${ }^{5}$ énonce la gratuité du recours à l'interprète, ainsi que les dispositions à adopter s'il est dans l'incapacité d'effectuer sa tâche. II mentionne également le serment liminaire "d'apporter son concours à la justice en son honneur et en sa conscience» que l'interprète doit prêter pour exercer légitimement son activité à la Cour. Si ces mêmes catégories d'honneur et de conscience se retrouvent dans la prestation de serment de l'interprète auprès des tribunaux et de la police, le serment prononcé devant la Cour est exclusif à cette juridiction.

Cette tension entre les caractéristiques de l'activité de l'interprète et leur inscription dans le contexte singulier de la procédure d'asile se manifeste aussi au niveau des injonctions déontologiques.

C'est ainsi que l'OFPRA, en plus des dispositions juridiques régissant l'interprétation en son sein, promeut une "charte de

5. Code de l'entrée et du séjour des étrangers et du droit d'asile, article R733-17. 
l'interprétariat ${ }^{6} »$, conditionnant le «bon exercice» de sa «mission de protection» à sa «relation de confiance»avec «les prestataires et les interprètes qu'ils emploient». On y retrouve les catégories normatives les plus fondamentales de l'interprétariat: la précision et la neutralité, c'est-à-dire l'obligation de n'être ni partiel ni partial.

De plus, un certain nombre d'exigences pratiques sont formulées dans ce document, notamment une fonction que l'on pourrait appeler «didascalique» de l'interprète, ce dernier étant tenu d'annoncer les modalités du discours qu'il rapporte. Il doit par exemple préciser qu'il procède à la reformulation d'un élément mal compris par le demandeur avant de procéder effectivement à cette reformulation.

De cet aperçu des cadres normatifs que l'administration de l'asile fixe à l'activité de l'interprète émerge donc une appréciation paradoxale du rôle de ce dernier, à la fois corrélé à la singulière complexité de la procédure de demande d'asile et contraint de se restreindre à une expertise impartiale purement linguistique.

\section{Le secteur de l'interprétariat professionnel}

Or on peut mettre en regard cette institutionnalisation de l'interprétariat avec sa réalité socioprofessionnelle.

Alors que le professionnalisme de l'interprète est mis en avant, comme garantie du respect des exigences de neutralité et d'impartialité, on remarque que le secteur connaît, paradoxalement, un défaut de professionnalisation. En décrivant cet état de fait, je ne mets pas en cause le professionnalisme des interprètes euxmêmes, mais j'interroge la façon dont la configuration socioprofessionnelle du secteur de l'interprétariat rapportée aux exigences de la procédure d'asile crée une situation d'interprétation originale et problématique.

Les interprètes sont en effet recrutés en fonction de besoins variables étroitement liés aux flux migratoires. Ils n'ont pas tous eu une expérience préalable ni une formation spécifique en interprétariat. En effet, ce sont les agences d'interprétariat prestataires qui entérinent la compétence de l'interprète à exercer 
sa fonction à l'OFPRA et à la CNDA, et qui doivent lui assurer des formations régulières. Or, dans un contexte très concurrentiel, et face aux nouveaux besoins en interprètes dans des langues dites "rares», ces structures ne peuvent pas toujours assurer ce suivi. À la CNDA, si les assesseurs et les rapporteurs bénéficient de mises au point régulières sur la situation des pays particulièrement stratégiques dans les parcours migratoires, les interprètes en sont exclus, et les institutions ne prévoient aucune formation spécifique pour eux.

Les connaissances juridiques et géopolitiques de l'interprète, nécessaires à l'exercice de son activité, ont ainsi comme seules sources son expérience et sa curiosité personnelle (souvent stimulée par un intérêt pour ces questions et par le souci de toujours être à la hauteur de la tâche). Elles ont pourtant une importance cruciale: si l'interprète ne connaît pas les lieux, les groupes ou les partis politiques dont parle le demandeur, il pourra difficilement traduire son récit de façon fidèle et crédible.

Les institutions entretiennent donc une ambiguïté majeure en insistant sur le caractère essentiel de ces connaissances, tout en se déchargeant de la responsabilité de les assurer.

De plus, selon des témoignages recueillis, la prestation de serment devant la CNDA a parfois lieu alors même que l'interprète y travaille depuis un certain temps: cet acte censé certifier l'intégrité de l'interprète est ainsi rendu secondaire, voire anecotique.

L'interprétariat dans le champ de l'asile se caractérise donc par une dichotomie entre sa définition très stricte par les institutions et la professionnalisation réelle du secteur ${ }^{7}$.

Ce constat n'induit pas un manque de compétence de la part des agences ou des interprètes, et ne doit pas masquer la grande diversité de profils parmi ces derniers (depuis le débutant mettant à profit son plurilinguisme jusqu'à l'interprète chevronné qui exerce son activité dans plusieurs secteurs en même temps). II révèle en revanche la place problématique réservée à l'interprétation au sein de la procédure d'asile, à la fois extrêmement normée et occultée dans sa dimension pratique. 


\section{La voix de l'interprète: enjeux politiques de la médiation}

C'est cette place paradoxale attribuée à l'interprétation qui permettra d'en clarifier les enjeux. La dialectique entre la norme et la liberté est en effet constitutive de l'interprétation ellemême, en ce que l'interprète est toujours situé dans un cadre conflictuel, ne serait-ce qu'au niveau sémantique, pour recréer un sens d'une langue à une autre. L'interprète, contrairement à sa représentation dans la "théorie du conduit», n'est en aucun cas réduit à une fonction purement instrumentale de transmission neutre d'un message d'une langue source à une langue cible. Il est au contraire pleinement intégré à la relation de communication dont il permet le déploiement. À première vue, une telle implication de l'interprète semble pourtant contredire l'exigence de neutralité qui est le fondement même de son éthique et de son efficacité professionnelles. C'est pourquoi il convient de revenir sur la signification réelle de la neutralité dans la dynamique d'interprétation; à cette condition, l'interprète peut être envisagé non plus comme un passeur, mais comme un médiateur, et on peut distinguer les enjeux réels de son activité dans la procédure de demande d'asile.

\section{Vers une notion critique de la neutralité}

La neutralité apparaît comme la valeur cardinale de l'interprète, parce qu'elle exprime sa loyauté à l'égard à la fois du message et de la situation: il n'est ni partiel ni partial.

Or cette acception de la neutralité est insuffisante, puisque message et situation ne sont pas deux entités distinctes, mais existent de manière concomitante. Dans le contexte de la procédure d'asile, l'interprète se voit en effet attribuer un rôle, qu'il jovera d'une certaine façon selon une multitude de configurations possibles ${ }^{8}$. Ce rôle d'intermédiaire le place au cœur de la rencontre asymétrique entre le demandeur et le fonctionnaire et l'implique nécessairement comme acteur de l'interaction.

8. Notamment la relation de confiance établie ou non avec l'officier de protection ou la formation de jugement; la possibilité que les autres acteurs parlent également les deux langues interprétées; l'attitude du demandeur à son encontre en fonction de divers critères (origine, genre, etc.). 
Le caractère inédit de chaque interaction crée pour l'interprète un positionnement tout aussi inédit, l'obligeant à adapter sa manière de traduire. Ainsi, si plusieurs interprètes remarquent que les exigences et consignes d'interprétation ne sont pas les mêmes à l'OFPRA et à la CNDA', ils notent que, au-delà de ces tendances générales, les façons d'interpréter diffèrent selon chaque interaction, et que "chaque interprète a son style», qu'il conserve malgré tout.

Il faut donc comprendre que la neutralité de l'interprète ne réside pas dans son hétérogénéité radicale, mais au contraire dans sa nécessaire participation à l'interaction en jeu.

Ainsi, même le modèle de l'interprétation «mot à mot», qui prétend attribuer à l'interprète un rôle purement technique, suppose son implication. Une interprète rapporte ainsi être particulièrement mal à l'aise lorsqu'à l'OFPRA, on lui demande de traduire mot à mot: ayant l'impression d'agir comme une enquêtrice lors d'un interrogatoire policier, elle se sent mise à une place qui n'est pas la sienne. Elle tente alors, par son ton, son rythme de parole, ou son attitude physique, de se détacher d'une posture inquisitrice et soupçonneuse, pour bien établir sa position d'intermédiaire et ainsi permettre la poursuite de l'interaction. Le cadre de l'interprétation mot à mot, loin de la neutraliser comme actrice de l'interaction, la pousse au contraire à s'adapter à la contrainte afin de pouvoir faire son travail, c'est-à-dire instaurer et maintenir une rencontre entre les deux autres acteurs.

Cet exemple montre que l'interprète, pour atteindre la réelle neutralité de sa fonction, ne se détache pas de l'interaction afin de faire office de conduit linguistique, mais doit au contraire s'y adapter et donc s'y investir.

Ainsi, l'interprète n'est pas un passeur, mais un médiateur, qui utilise des ressources extralinguistiques pour assurer sa neutralité, c'est-à-dire veiller à ne pas se ranger du côté de l'un ou l'autre acteur de l'interaction, et ainsi respecter les normes déontologiques de sa profession. Il faut donc changer de focale: il ne s'agit pas de la mise en présence de deux interlocuteurs qui émettent et reçoivent des messages par le truchement d'un

9. Selon les mots d'une interprète, dans le premier cas, le format de l'entretien retranscrit semble appeler plutôt une interprétation "verbatim», en reproduisant même les erreurs et scories du discours du demandeur; dans le second cas, les juges «veulent seulement des réponses à leurs questions» et laissent une certaine latitude à l'interprète. 
interprète, mais d'une «rencontre médiatisée par interprète ${ }^{10}$ ». Dès lors, le rôle de l'interprète devient particulièrement intéressant et crucial dans l'appréhension des rapports de pouvoir fortement inégaux, caractéristiques des interactions entre ceux qui requièrent et ceux qui octroient l'asile politique.

Interroger les enjeux politiques de l'interprétation dans la procédure de demande d'asile, selon les conditions indiquées, ce n'est bien sûr pas prétendre déterminer l'influence de l'interprète dans l'acceptation ou le refus d'une demande. La question de la motivation est essentielle, tant au niveau du demandeur présentant sa requête qu'à celui de l'administration justifiant sa décision selon des critères juridiques. Mais le processus de décision est extrêmement complexe et dépasse peut-être même le cadre des entretiens à l'OFPRA ou des audiences à la CNDA.

Pourtant, les «rencontres médiatisées par interprète» qui s'y déroulent ont bien des enjeux proprement politiques.

\section{L'interprétation ou la parole politique}

De quel côté l'interprète se tient-il? Est-il indirectement un agent de l'administration qui le rémunère (indirectement), le surveille et lui dicte ses normes de travail? Ou bien est-il l'allié du demandeur, lui donnant la parole qu'il ne peut pas prendre, proche de lui par le partage d'une langue et de référents culturels? S'il est un simple outil de transcodage linguistique, la question est évacuée, masquée par l'acception réductrice de la neutralité. Mais en tant que médiateur, l'interprète est pleinement inclus dans le rapport de pouvoir qui se jove entre le demandeur qui doit convaincre et le fonctionnaire qui doit décider.

La participation de l'interprète n'est pas une implication personnelle: l'âge, l'ancienneté, le parcours de ceux que j'ai rencontrés sont très variés, ainsi que leur sensibilité à la problématique de l'asile, mais tous sont attentifs à ne pas faire entrer dans leur travail leurs opinions ou convictions personnelles. Exception faite des cas très marginaux et spécifiques parfois évoqués dans les médias", les interprètes n'agissent jamais selon leur intérêt personnel et font leur travail sans privilégier ni discriminer tel

10. Concept issu de la théorie interactionniste de l'interprétation de Wadensjö (1998).

11. Le Monde du 22 février 2016. Ces allégations, qui ont suscité de vives réactions dans le secteur de l'interprétariat, n'ont pas été totalement avérées et doivent donc être envisagées avec prudence. 
ou tel dossier. Mais notre analyse de la neutralité révèle que, si l'interprète n'est pas impliqué en tant que personne dans l'interaction, il l'est en tant que sujet interprétant, puisque c'est sur lui que repose la rencontre et par lui qu'elle se déploie.

Il faut donc envisager l'interprète non comme une individualité, mais comme une subjectivité à l'œuvre dans le dispositif de la demande d'asile, et en tenir compte pour comprendre les rapports de pouvoir qui s'exercent alors.

La situation suivante observée à la CNDA permet d'illustrer ce point: le président de la formation de jugement pose une série de questions à un couple de requérants sur l'évènement qui, selon eux, a motivé leur départ et justifie leurs craintes. L'échange est serein, les requérants se voyant attribuer un temps de parole assez long et l'interprète traduisant leurs propos par séquences, en précisant à chaque fois qu'elle doit les interrompre pour traduire au fur et à mesure, ce qu'ils acceptent sans protester. Soudain, le président se met à poser des questions de plus en plus précises et lapidaires, accélérant le rythme de l'échange, puis annonce, de manière assez emphatique, "J'arrive à présent au terme de mon raisonnement», avant de suspendre son discours. Après quelques secondes de silence, et plusieurs regards insistants à l'interprète, celle-ci comprend que le président souhaite qu'elle traduise cette formule isolément, pour conférer un effet rhétorique aux conclusions qui vont suivre, et qui mettent en doute le récit des requérants. Mais ce temps de latence a désamorcé toute l'efficacité rhétorique de la formule, qui tombe à plat, au point que le président déplorera peu après, sur un ton ironique, que les requérants ne parlent pas français.

Cette séquence montre que l'interprète, loin d'avoir manqué de réactivité, a au contraire pleinement joué son rôle et respecté sa neutralité, en maintenant l'égalité des paroles qu'elle avait instaurée. Le fait qu'elle ne suive pas le président dans l'offensive rhétorique visant à décrédibiliser les requérants n'est donc pas un manquement de sa part, mais au contraire la preuve de son professionnalisme: elle ne s'associe aux stratégies d'aucune des deux parties dans sa confrontation avec l'autre et montre ainsi qu'elle n'est présente ni pour convaincre ni pour mettre en doute, mais pour créer un espace sémantique commun.

Une approche critique de la neutralité de l'interprète permet donc de mieux cerner son rôle réel de médiateur, et les efforts et stratégies qu'il déploie. Ce rôle est d'autant plus crucial qu'il 
touche en son cœur la question de la véracité des propos des demandeurs, si déterminante dans la procédure d'asile, mais aussi dans l'économie morale ${ }^{12}$ de la suspicion à leur égard.

La liberté inhérente à l'acte d'interprétation, régulée et renégociée en permanence, dessine un modèle original d'agentivité de l'interprète comme intermédiaire. Ce faisant, elle révèle l'importance capitale de ce dernier au sein des confrontations radicalement politiques du dispositif de l'asile.

En configurant ces rencontres, il est le seul à faire entendre la complexité politique et humaine des voix de l'asile.

maxime.marechal@ ehess.fr

Maxime Maréchal a suivi un master de philosophie à l'Université Paris 4. Son mémoire, portant sur la théorie du symbole chez Paul Ricœur, I'a conduit à étudier la traduction selon la tradition philosophique de l'herméneutique. Volontaire en service civique au sein de la "mission migrants» du Samu social de Paris où il a découvert l'interprétariat de service public, il a décidé d'approfondir sa compréhension des problématiques liées à la demande d'asile en s'inscrivant en master à l'École des hautes études en sciences sociales (EHESS).

SOURCES CITÉES

AKOKA Karen, «L'archétype rêvé du réfugié», in Plein droit, 3, 2011, p. 13-16.

BELORGEY Jean-Michel, «Quand l'exil s'interprète mal», in Plein droit, 3, 2013, p. 24-26.

FASSIN Didier, BOUAGGA Yasmine et al., Juger, réprimer, accompagner. Essai sur la morale de l'État, Paris, Éditions du Seuil, 2013.

FOUCAULT Michel, L'Ordre du discours, Paris, Gallimard, 1970.

HAUCHARD Amaury et CHARNET Agathe, «La vie volée d'un demandeur d'asile érythréen", Le Monde, article en ligne mis à jour le 22 janvier 2016, consulté le 13 mai 2019.

LAACHER Smaïn, Croire à l'incroyable. Un sociologue à la Cour nationale du droit d'asile, Paris, Gallimard, 2018.

LARCHET Keltoume et PÉLISSE Jérôme, «Une professionnalisation problématique: les experts judiciaires interprètes-traducteurs ", in Formation emploi, 108, 2009, p. 9-24.

LEANZA Yvan et BOIVIN Isabelle, «Interpréter n'est pas traduire. Enjeux de pouvoir autour de l'interprétariat communautaire», actes du colloque L'éducation en contextes pluriculturels: la recherche entre bilan et prospective, 2008, www.researchgate.net/

12. La «production, la circulation et l'appropriation des valeurs» dans un groupe social donné (Fassin et al., 2013). 
publication/242165999_Interpreter_n'est_pas_traduire_Enjeux_de_pouvoir_autour_ de_l'interpretariat_communautaire, consulté le 13 mai 2019.

POINTURIER Sophie, Théories et pratiques de l'interprétation de service public, Paris, Presses Sorbonne Nouvelle, 2016.

VALLUY Jérôme, « La fiction juridique de l'asile», in Plein droit, 4, 2004, p. 17-22.

WADENSJÖ Cecilia, Interpreting as Interaction, Londres, Routledge, 1998. 